\title{
Debatedor
}

\section{Modelo de desenvolvimento, agrotóxicos e saúde: prioridades para uma agenda de pesquisa e ação}

Development model, pesticides, and health: Priorities for research and action agenda

Neice Müller Xavier Faria ${ }^{1}$

O Brasil está num momento de grande desenvolvimento econômico com indicadores consistentes de crescimento, com destaque para o agronegócio, que apresenta projeções de crescimento expressivo (BRASIL, 2011a).

O artigo de Porto e Soares, abordando o panorama da realidade agrícola brasileira e propondo uma agenda de pesquisa em relação ao Modelo Desenvolvimento, Agrotóxicos e Saúde é de fundamental importância por abordar um tema de enorme impacto social, econômico e ambiental. Os autores trazem uma reflexão sobre a trajetória da realidade agrícola brasileira a partir da "Revolução Verde", que se consolidou no atual modelo agroexportador, e destacam os efeitos deste modelo sobre a saúde, em especial dos trabalhadores rurais.

Os problemas decorrentes do uso de agrotóxicos envolvem contaminações através de resíduos em alimentos, contaminações ambientais, desinsetizações e exposição ocupacional. O uso de agrotóxicos tem sido relacionado a vários problemas de saúde entre agricultores (FARIA et al., 2004; ARAUJO et al., 2007; FARIA; ROSA; FACCHINI, 2009). A questão em debate é qual o modelo de desenvolvimento desejável no setor produtivo agrícola levando em conta não apenas o ganho financeiro imediato, mas também a condição de vida da população e a sustentabilidade deste modelo de desenvolvimento.

Nos últimos anos, o Brasil avançou em muitos aspectos nas políticas públicas em relação a agrotóxicos e saúde. Um exemplo é o Programa de Análises de Resíduos de Agrotóxicos - PARA (ANVISA, 2009), que é hoje uma referência internacional em relação ao controle de resíduos em alimentos, embora ainda necessite de aperfeiçoamento.

Este texto traz uma contribuição para o debate sobre agrotóxicos e saúde, apresentando propostas direcionadas à saúde dos trabalhadores rurais. A partir de questões que emergiram de estudos epidemiológicos, bem como de debates envolvendo profissionais de saúde e da área agrícola, pesquisadores e agricultores, foram estruturadas propostas para uma agenda de pesquisa e de ações, organizadas em sete blocos temáticos.

\section{As informações sobre consumo de agrotóxicos}

Embora seja o maior consumidor mundial de agrotóxicos, o Brasil continua, até o momento, sem um sistema de informações que monitore o consumo de agrotóxicos no país. A única fonte oficial é o Censo Agropecuário, segundo o qual em média 30\% dos estabelecimentos rurais brasileiros costumavam usar agrotóxicos (INSTITUTO BRASILEIRO DE GEOGRAFIA E ESTATÍSTICA, 2006). Este percentual é mais baixo do que o encontrado em estudos populacionais, nos quais a maioria dos agricultores relata uso frequente destes produtos (FARIA et al., 2004; ARAUJO et al., 2007; FARIA; ROSA; FACCHINI, 2009). Além disso, o censo não tem captado aspectos importantes como os tipos químicos ou a quantidade usada.

\footnotetext{
${ }^{1}$ Médica do Trabalho. Doutora em Epidemiologia. Setor de Vigilância à Saúde da Secretaria Municipal de Saúde de Bento Gonçalves, RS. Pesquisadora associada ao Grupo de Pesquisas em Saúde do Trabalhador, Universidade Federal de Pelotas, Pelotas, RS, Brasil. Contato: Rua República, 80/1.401, Cidade Alta, Bento Gonçalves, RS, Brasil, CEP: 95700-000. E-mail: neice@clinicagianisella.com.br
} 
Na prática, não se sabe o que é prescrito através do Receituário Agronômico (RA), nem o que é vendido oficialmente em lojas agrícolas e muito menos o que é adquirido pelo comércio informal/contrabando. Isso coloca o Brasil como refém dos interesses da indústria, visto que não existem meios de monitorar problemas para definir prioridades de ações.

No Rio Grande do Sul houve uma experiência que avaliou aproximadamente 95\% dos RAs de 1996. O banco de dados, com 217.000 receituários, foi examinado para um estudo epidemiológico, sendo verificadas importantes limitações, com destaque para a grande proporção de dados ignorados/ ilegíveis. Além disso, mesmo nos receituários com dados completos, observou-se que não representavam a realidade agrícola conforme profissionais da área agrícola e conforme um estudo feito pelo Instituto Brasileiro de Geografia e Estatística (IBGE) (FARIA; FASSA; FACCHINI, 2007). Os autores recomendaram aprofundar a discussão visando a estruturar um sistema de informações sobre o uso de agrotóxicos no Brasil.

Um dos caminhos seria revitalizar o RA, conforme seus objetivos originais, disponibilizando os dados para análises, a exemplo do que hoje é feito com os dados oficiais de saúde. Considerando a tecnologia atual, a alimentação deste sistema poderia ser feita on line, no momento da venda do produto. Seria um caminho mais simples, uma vez que o RA é obrigatório, já está implantado (apesar de alguns desvios) e tem as responsabilidades bem definidas na legislação sobre agrotóxicos (Lei Federal $\mathrm{n}^{\mathrm{o}}$ 7.802/1989).

Outro caminho seria utilizar dados de notas fiscais da venda deagrotóxicos. Uma experiêncianeste sentido está ocorrendo no Mato Grosso, através do Instituto de Defesa Agropecuário (Indea). Este sistema informatizado utiliza notas fiscais acrescentando dados do receituário agronômico. Atualmente, as informações estão sendo analisadas, mas ainda não existem resultados publicados avaliando esta experiência.

Nenhuma das opções acima incluiria os agrotóxicos adquiridos por contrabando, que envolvem um volume considerável de produtos, muitas vezes mais tóxicos que os produtos registrados, como, por exemplo, o arsênico, usado como formicida em cerca de $20 \%$ das propriedades na fruticultura do Rio Grande do Sul (FARIA; ROSA; FACCHINI, 2009) e cujo uso não seria captado por RA ou notas fiscais.

A estruturação de um sistema de informações sobre o consumo de agrotóxicos é uma prioridade como política pública brasileira e como base de dados para futuras pesquisas. A proposta deve incluir a divulgação de análises periódicas, disponibilizando o banco de dados de forma a subsidiar pesquisas e ações de proteção à saúde e ao meio ambiente.

\section{As informações sobre intoxicações por agrotóxicos}

Além da ausência de registros referentes à exposição a agrotóxicos, existem muitas limitações nas informações dos efeitos dos agrotóxicos sobre a saúde. A escassez de informações que permitam monitorar as condições de saúde no trabalho agrícola já foi apontada por diversos autores (OLIVEIRA-SILVA; MEYER, 2003; FARIA; FASSA; FACCHINI, 2007). Os registros oficiais são bastante limitados e em geral referem-se apenas às intoxicações agudas por agrotóxicos.

Uma iniciativa governamental para resolver este problema é a notificação através do Sistema de Agravos de Notificação - Sinan (BRASIL, 2008). Atualmente, as intoxicações por agrotóxicos são um dos agravos de notificação compulsória para todos os serviços de saúde, conforme Portaria no 104/MS/2011, Anexo I (BRASIL, 2011b). Além do Sinan, historicamente o principal registro de intoxicações por agrotóxicos tem sido o Sistema Nacional de Informações Tóxico-Farmacológicas (Sinitox), que abrange uma rede nacional de 35 Centros de Informação e Assistência Toxicológica (Renaciat).

A diferença entre os dois sistemas de informações é bem relevante. Uma avaliação das informações existentes no SUS mostrou que, em 2006, foram notificados 3.208 casos de intoxicação pelo Sinan e 15.783 pelo Sinitox (BRASIL, 2008). Como os centros da Renaciat prestam assistência toxicológica, o Sinitox consegue captar muitos casos atendidos em serviços de urgência, especialmente os de maior gravidade.

Por outro lado, o Sinan tem captado proporcionalmente mais casos de intoxicações por agrotóxicos relacionadas ao trabalho. Considerando os 4.524 casos registrados pelo Sinan em 2007, 24,6\% eram relacionados ao trabalho. $\mathrm{O}$ percentual relativo foi o dobro do registrado pelo Sinitox em 2006, que captou 12,2\% (dos 15.783 registros) como circunstâncias ocupacionais (BRASIL, 2008). A subnotificação mais acentuada dos casos de intoxicações por agrotóxicos relacionadas ao trabalho foi verificada em um serviço municipal de vigilância à saúde com busca ativa dos casos de intoxicação por agrotóxicos em prontuários de atendimentos de urgência (FARIA; FASSA; FACCHINI, 2007). No período relatado (2002 a 2004), foi verificada uma incidência de 61,5 casos/100.000 habitantes, bem maior que os 8,0/100.000 encontrados nos dados nacionais do Sinitox no mesmo período. Além disso, a maioria dos casos era ocupacional, contrastando com os registros do Sinitox (nacional e estadual), nos quais predominavam casos acidentais e intencionais. Atualizando a informação do referido serviço para o triênio 2006-2008, observou-se uma incidência média de 56,3 casos anuais por 100.000 habitantes. E a proporção de intoxicações relacionadas ao trabalho se manteve bem maior que as demais circunstâncias: 54,9\% de casos ocupacionais; 30,3\% 
de acidentais e 14,9\% de intencionais (BENTO GONÇALVES [município], 2009).

Outra fonte oficial de informações é o Censo Agropecuário do IBGE (INSTITUTO BRASILEIRO DE GEOGRAFIA E ESTATÍSTICA, 2006), que encontrou 25 mil estabelecimentos rurais que informaram ter pelo menos uma pessoa intoxicada por agrotóxicos em 2006. Calculando 25 mil pessoas intoxicadas/ total de pessoas ocupadas nos estabelecimentos rurais (16.567.544), teríamos uma estimativa bruta de 151 pessoas intoxicadas por 100 mil trabalhadores rurais por ano, bem maior que o estimado por qualquer sistema de informação anteriormente mencionado.

Estes dados confirmam uma das prioridades em uma agenda tanto de pesquisa, como de vigilância em saúde: qualificar as equipes de saúde (em especial os serviços de urgência e as equipes de unidades de saúde de área rural) como estratégia para melhorar o diagnóstico, o manejo dos casos agudos e estimular o registro de casos de intoxicação. A estratégia de busca ativa se apresenta como uma maneira interessante e viável de melhorar o registro dos casos não notificados. E a devolução periódica dos resultados pode estimular a adesão por parte dos profissionais de saúde.

Por outro lado, a identificação e o registro de doenças crônicas relacionadas aos agrotóxicos permanecem como um enorme desafio, sendo ignorada a dimensão destes problemas no Brasil. Em diagnósticos como câncer, hepatopatias, neuropatias, distúrbios hormonais e outros, o estabelecimento de nexo causal é bastante difícil devido à complexidade de fatores que podem interferir na evolução da doença. Em geral, a relação com agrotóxicos é estabelecida em estudos com amostras representativas, sendo, neste caso, um nexo epidemiológico. Mas esta é uma lacuna que merece ser contemplada com uma agenda de pesquisas, com intervenções específicas, para que seja possível melhorar o reconhecimento dos casos.

\section{O diagnóstico de intoxicação aguda por agrotóxicos}

Uma dificuldade histórica, em vários países incluindo o Brasil, tem sido a falta de uma padronização dos critérios para definição de casos de intoxicações agudas por agrotóxicos. Buscando solucionar esta questão, um grupo internacional de experts ligado à Organização Mundial de Saúde (OMS) elaborou uma proposta de classificação utilizando uma matriz que leva em conta os critérios de exposição, efeitos sobre a saúde e causalidade (THUNDIYIL et al., 2008). Esta proposta já foi utilizada no Brasil em um estudo na fruticultura da Serra Gaúcha (FARIA; ROSA; FACCHINI, 2009) e está atualmente sendo testada em área de fumicultura (estudo em andamento).

A proposta da OMS leva em conta a toxicologia conhecida do produto e avalia os casos conforme gravidade: leve, moderada e severa. Por isso inclui os ca- sos ocupacionais que, embora sejam mais frequentes, costumam apresentar gravidade menor que os casos intencionais (tentativas de suicídio e suicídios). E os casos leves e moderados nem sempre procuram os serviços de saúde (principalmente em locais com dificuldade de acesso ou épocas com ritmo intenso).

Uma vantagem adicional é que a proposta da OMS permite uma definição de caso possível ou provável sem depender da disponibilidade de exames toxicológicos específicos, que em geral não estão disponíveis na maioria dos serviços. Além disso, a padronização do critério de gravidade facilita no treinamento de profissionais de saúde de serviços de urgência e nos registros de saúde.

Contudo, esta proposta necessita ser avaliada em estudos epidemiológicos realizados em diferentes contextos agrícolas, bem como adaptada para ser usada nas diversas regiões do país. Na proposta de classificação de intoxicações agudas, a tabela 1 descreve os efeitos sobre a saúde relacionados conforme os principais tipos químicos (THUNDIYIL et al., 2008). Embora os tipos apresentados representem a maior parte dos produtos usados na agricultura, já foram identificados outros tipos químicos de uso frequente que não estão incluídos na referida tabela. Entre eles podem ser citados: fungicidas mancozeb, metalaxil, captana; inseticidas neonicotinoides (imidacloprido), fipronil e sulfluramida; herbicidas clomazona e sulfentrazona ou reguladores de crescimento cianamida e flumetralina. Estes e outros agrotóxicos que venham a ser identificados também devem ser incluídos numa proposta brasileira, atualizando a tabela original e usando a mesma metodologia.

Como esta metodologia ainda é pouco conhecida pelos profissionais de saúde, uma agenda de prioridades deve incluir a ampliação da proposta em relação aos tipos químicos e a capacitação de profissionais de saúde para utilização deste instrumento proposto pela OMS. Recomenda-se também a realização de estudos epidemiológicos usando esta metodologia padronizada para avaliar a dimensão das intoxicações agudas nas diversas regiões do país.

\section{Capacitação dos profissionais de saúde e educação}

Atualmente tem crescido bastante a expansão de serviços de saúde em área rurais, em boa parte dos casos através da Estratégia de Saúde da Família (ESF). A proposta de atuação das equipes de ESF prevê contato direto frequente entre as pessoas da área de abrangência e as equipes de saúde. Assim, uma abordagem essencial seria capacitar as equipes de ESF, em especial aquelas que atendem em área rural, para o reconhecimento e o manejo dos casos de intoxicação por agrotóxicos e também para difundir orientações visando a prevenir ou reduzir os efeitos da exposição aos agrotóxicos ou, ainda, estimular a discussão de uma mudança no modelo de produção agrícola, em uma 
abordagem integrada com a área de saúde do trabalhador e com profissionais de extensão rural.

Também seria recomendável a parceria com a educação, envolvendo projetos de capacitação de professores de escolas rurais sobre o tema dos agrotóxicos, ampliando o alcance das orientações para os futuros agricultores.

Outro grupo que necessita uma abordagem específica são os profissionais, em especial médicos, que atendem em serviços de urgência, uma vez que apresentam maior dificuldade de adesão às diversas formas de educação continuada na área da saúde. Boa parte dos casos de intoxicações agudas leves e moderadas que procuram os serviços de saúde não são reconhecidos como tal. Da mesma forma, casos de problemas crônicos reagudizados (como asma, alergias, tonteiras, cefaleias, hepatopatias e outros) recebem tratamentos sintomáticos sem nenhuma orientação sobre os riscos envolvendo o contato com agrotóxicos.

Quando questionados, a maioria dos profissionais de saúde admite sua limitação no assunto, considerado de grande complexidade e repleto de incertezas. O fato é que existe escassez de informações toxicológicas para os profissionais de saúde, principalmente em língua portuguesa. Ao mesmo tempo, a rapidez com que a indústria de agrotóxicos lança novos produtos no mercado é muito superior à dos pesquisadores em verificarem os riscos e divulgarem as informações. Enquanto boa parte das informações disponíveis está concentrada nos tipos químicos tradicionais, os estudos em campo realizados entre agricultores estão evidenciando uma variedade bem maior de tipos químicos já em uso rotineiro. Um exemplo disto é o inseticida fipronil, lançado no mercado americano em 1996 e que, em 2006, apenas 10 anos depois, já era usado pela maioria das propriedades na fruticultura na Serra Gaúcha (FARIA; ROSA; FACCHINI, 2009), embora seja pouco conhecido pelos pesquisadores.

Assim, uma proposta viável, de custo acessível e de extrema importância, seria criar um site com informações toxicológicas gratuitas, abordando efeitos agudos e crônicos, bem como orientações de manejo clínico para cada tipo químico de agrotóxicos usado no país. Uma experiência nesta direção existe nos Estados Unidos, onde uma rede de universidades americanas criou um site (Extoxnet ${ }^{2}$ ) que disponibiliza gratuitamente várias informações toxicológicas, organizadas de forma sintética, para consulta rápida, sobre a maioria dos tipos químicos. A implementação de uma proposta semelhante no Brasil, com suporte de profissionais da toxicologia e de outras áreas afins, tem potencial de ampliar a qualificação dos profissionais de todos os tipos de serviços, que poderiam inclusive retroalimentar o sistema informando novos produtos/sintomas clínicos detectados na prática clínica.

\section{Monitorizações biológicas de exposição e/ou efeitos dos agrotóxicos}

Em relação aos exames laboratoriais toxicológicos, uma rápida busca nos principais laboratórios do país, públicos e/ou privados, revela que, mesmo sem considerar os custos financeiros, existe uma enorme limitação na disponibilidade de exames para avaliar exposição ou intoxicação por agrotóxicos. A maioria dos laboratórios só realiza exames de colinesterase (plasmática ou, em alguns casos, colinesterase eritrocitária), que apresenta limitações como biomarcador que já foram apontadas em artigo anterior (FARIA; FASSA; FACCHINI, 2007). Além de ser influenciada por inúmeros fatores, existem suspeitas sobre o desenvolvimento de efeitos neurotóxicos persistentes, em trabalhadores ou bebês, após exposição prolongada e em "baixas" doses aos organofosforados, que poderiam ocorrer mesmo sem uma crise colinérgica clássica (RAY; RICHARDS, 2001; KEIFER; FIRESTONE, 2007). A definição do ponto de corte para a colinesterase, especialmente a plasmática, para monitorização biológica também tem sido objeto de controvérsias e deveria ser objeto de pesquisas específicas para tal definição (FARIA; FASSA; FACCHINI, 2007).

Além da colinesterase, com certa dificuldade, outros exames de monitorização biológica também podem ser encontrados: paraquat, organoclorados, cobre, arsênico e manganês. Outras opções de exames toxicológicos são extremamente raras ou são realizadas por laboratórios toxicológicos internacionais. A questão está nos outros grupos químicos também usados com frequência. Por exemplo: o tipo químico mais vendido no Brasil é o herbicida glifosato, um dos fungicidas mais usados é o mancozeb do grupo dos ditiocarbamatos. Ou seja, os exames laboratoriais disponíveis não avaliam boa parte dos produtos usados no país. Assim, um dos desafios óbvios é a ampliação da disponibilidade de outros indicadores biológicos.

Estudos internacionais vêm, há algum tempo, utilizando outros marcadores biológicos de vários agrotóxicos, com destaque para metabólitos urinários, que são mais adequados para trabalho de campo, em estudo rural e monitorização biológica de trabalhadores. No estudo americano de saúde na agricultura (AHS), foram estudados metabólitos urinários de atrazina, metolachlor, glifosato e chlorpirifós (CURWIN et al., 2007; THOMAS et al., 2010). A exposição ambiental de crianças a inseticidas foi avaliada através metabólitos urinários de cinco tipos de piretroides (LU et al., 2006) e metabólitos urinários de ETU (Etileno-Tio-Ureia), oriundos da exposição a fungicidas ditiocarbamatos. Foram avaliados em população geral do Reino Unido (JONES et al., 2009) e em trabalhadores da vitivinicultura na Itália (FUSTINONI et al., 2008).

Embora a informação referida seja considerada uma abordagem válida, têm sido evidenciadas discre- 
pâncias entre a informação referida de exposição e os resultados de metabólitos urinários dos agrotóxicos (PERRY; MARBELLA; LAYDE, 2006).

Conforme já foi apontado por outros autores:

os pontos críticos de muitos estudos epidemiológicos sobre problemas de saúde relacionados aos agrotóxicos são as limitações na avaliação da exposição, um pequeno número de indivíduos expostos e as dificuldades em estimar falhas na informação de exposição. (JUREWICZ; HANKE, 2006, p. 152. Tradução livre da autora)

A insuficiência de opções de exames de monitorização da exposição aos agrotóxicos é uma preocupação já identificada por outros autores (KEIFER; GASPERINI; ROBSON, 2010), incluindo países latinos como Chile (RIOS; SOLARI, 2010) e Colômbia (CARDENAS et al., 2005).

A complexidade do tema é ainda maior, pois os estudos brasileiros revelam que, em geral, a exposição ocupacional dos trabalhadores rurais é multiquímica, porém os estudos costumam testar o efeito toxicológico de um ingrediente ativo de cada vez. Na Serra Gaúcha, um estudo na fumicultura revelou que os trabalhadores usavam em média de 12 produtos diferentes na propriedade e, em alguns casos, mais de 20 produtos (FARIA; ROSA; FACCHINI, 2009). Como avaliar o efeito da exposição simultânea a tantos produtos químicos?

Avaliar a exposição aos agrotóxicos tem sido um desafio para vários pesquisadores. Pesquisadores da Carolina do Norte debateram a complexidade das exposições aos pesticidas entre trabalhadores rurais migrantes e temporários, em que a exposição não é prédefinida e onde existe uma rede de fatores interferindo na possibilidade de contaminação e nas estratégias de intervenção. Um dos aspectos avaliados foi a monitorização biológica (ARCURY et al., 2006; KEIFER; GASPERINI; ROBSON, 2010). Este grupo estudou trabalhadores rurais migrantes e sazonais que apresentavam exposições repetidas a múltiplos pesticidas. Foram examinados metabólitos urinários de 12 tipos de pesticidas e a maioria deles apresentou testes alterados para diversos metabólitos específicos (ARCURY et al., 2009; ARCURY et al., 2010), confirmando a importância de dimensionar a exposição multiquímica.

Portanto, a monitorização biológica da exposição multiquímica aos pesticidas é, sem dúvida, um grande desafio e uma prioridade para a agenda de pesquisa e para o planejamento de ações relativas a agrotóxicos e saúde.

\section{A proteção do trabalhador com exposição aos agrotóxicos}

A diversidade de fatores que determinam as consequências do uso de agrotóxicos é enorme a alguns deles apresentam um grande desafio, evidenciando a ideia simplista inserida na proposta do "uso correto e seguro" destes produtos (GARCIA; ALVES FILHO, 2005). A proposta completa, visando a reduzir a exposição química e os riscos de intoxicação devido ao uso de agrotóxicos, envolve uma abordagem mais ampla com identificação dos riscos, definição das medidas de controle em cada situação, implementação de medidas de proteção coletiva (incluindo controle dos riscos na fonte ou no processo de produção) e, só então, as medidas de proteção individual (ALVES FILHO, 2001; GARCIA; ALVES FILHO, 2005). Estas incluem práticas de segurança, medidas de higiene no trabalho, formas diversas de educação para a saúde e segurança no trabalho e o uso correto de Equipamentos de Proteção Individual (EPI), que devem ser adequados ao risco específico.

As dificuldades na implementação de um Programa de Segurança e Saúde no Trabalho são diversificadas, incluindo a escassez de técnicos com formação na área de saúde ocupacional atuando em área rural. Com frequência, as orientações técnicas de proteção no trabalho com agrotóxicos são restritas ao uso genérico de EPIs, sem avaliação da situação de risco.

Existem algumas dificuldades que devem ser reconhecidas, como, por exemplo, as orientações de lavar-se logo após terminar os trabalhos envolvendo contato direto com agrotóxicos ou lavar as roupas contaminadas antes de uma nova utilização são consensuais como medidas de proteção entre pesquisadores, técnicos e trabalhadores rurais. Contudo, em algumas situações, a exposição química ocorre em locais de trabalho sem disponibilidade de água limpa para higiene corporal. A questão é particularmente crítica em regiões brasileiras onde existe escassez de água durante boa parte do ano. Ou seja, nestas situações, o problema de higiene ultrapassa o risco químico no trabalho e torna-se uma questão sanitária de solução mais ampla.

A polêmica é mais acentuada quando o assunto é o uso de EPIs. O uso de EPIs deveria ser uma medida complementar dentro de um Programa de Segurança e Saúde no Trabalho - PSST (ALVES FILHO, 2001). No entanto, em boa parte dos treinamentos para trabalhadores rurais, a abordagem de prevenção de intoxicações é centrada no uso de EPIs, padronizando uma indicação genérica, sem levar em conta a situação de trabalho.

Considerando que a boa parte dos agrotóxicos são absorvidos através da pele do trabalhador (THUNDIYIL et al., 2008; VITALI et al., 2009; FARAHAT et al., 2010; NGO; O'MALLEY; MAIBACH, 2010), a proteção corporal completa seria fundamental para reduzir a absorção de agrotóxicos e o uso de EPIs adequados reduziria o risco de intoxicações (GARCIA; ALVES FILHO, 2005; CATANO et al., 2008; VITALI et al., 2009). Os problemas com uso inadequado de EPIs são frequentes, apesar do aumento dos treinamentos direcionados aos trabalhadores rurais. Por razões diversas, incluindo custos, alguns trabalhadores optam por improvisar EPIs que podem aumentar a contaminação quí- 
mica, como uso de lenços ou máscaras de tecido para substituir respiradores com filtro químico.

Mesmo usando corretamente EPIs indicados, alguns agricultores queixaram-se que, ao final de algumas horas de aplicação de agrotóxicos, a roupa de proteção, com revestimento hidrorrepelente, muitas vezes ficava molhada na região posterior das coxas, próximo do assento do trator (sem cabine). A queixa dos agricultores é apoiada por estudos que também verificaram problemas limitando a eficácia dos EPIs (OLIVEIRA; MACHADO NETO, 2005; BALDI et al., 2006; VEIGA et al., 2007). Além disso, em certas tarefas específicas, como no raleio da fruticultura (remoção de alguns frutos para fortalecer os demais que permanecem na planta), a queixa era de que as luvas recomendadas para uso de agrotóxicos atrapalhavam a tarefa, que exige habilidade e precisão de movimentos. E reivindicaram a necessidade de EPIs específicos para estas tarefas.

Este tema vem sendo debatido por pesquisadores da Fundacentro (GARCIA; ALVES FILHO, 2005), que abordaram a controvérsia envolvendo os EPIs, criticando a simplificação, a generalização e o reducionismo em relação à indicação técnica e ao alcance do uso da proteção individual. Consideram que estes fatores levam à uma mistificação das recomendações e ao descrédito do usuário em relação ao uso, alimentando-se, assim, as reações de negação ou desprezo sobre a existência do risco. Além disso, segundo Alves Filho (2001), uma recomendação de uso de EPI pode ser entendida como solução final para a segurança do aplicador. Isso poderia criar uma falsa sensação de segurança, estimulando atitudes de exposição exagerada ao risco.

Uma sugestão, apresentada por Kissmann (2001), é profissionalizar a aplicação de agrotóxicos, restringindo as atividades de maior risco (aplicação e preparo da calda) a profissionais treinados (aplicadores com certificação) e devidamente protegidos. Esta proposta pode ser adequada para grandes empresas agrícolas, mas é difícil de ser implantada nos estabelecimentos menores da agricultura familiar, que respondem pela maioria dos trabalhadores rurais brasileiros.

Alguns profissionais sugeriram formas de viabilizar acesso a crédito para compra EPIs como estratégia de reduzir os problemas de intoxicações por agrotóxicos. A proposta seria acompanhada de treinamentos sobre segurança química no trabalho. Embora treinamentos sejam recomendáveis de uma forma geral, a estratégia de estimular a aquisição de EPIs mantém o foco central das ações de proteção no uso de EPIs e poderia esbarrar na maior resistência ao uso dos EPIs, que é o conforto térmico já apontado em estudos anteriores (SOARES; FREITAS; COUTINHO, 2005; VEIGA et al., 2007).

Quase todos os trabalhadores rurais se queixam do desconforto dos EPIs como sendo o maior obstáculo ao uso destes equipamentos (principalmente máscaras e roupas impermeáveis para proteção da pele). Embora as restrições econômicas possam contribuir para diminuir o uso de EPIs, conforme relato de vários agricultores, a questão principal é o desconforto, principalmente térmico. A queixa de desconforto vale para todos os tipos de equipamento de aplicação, com exceção do trator com cabine fechada, ar-condicionado e filtro químico. Essa queixa inclui quem mora em regióes quentes e regiões mais frias, pois a época de uso intensivo é sempre a de temperaturas mais elevadas. O questionamento de muitos é por que os EPIs ainda são tão desconfortáveis numa época com tanta tecnologia disponível.

Assim, as questões que emergem para uma agenda de pesquisa referem-se à qualidade dos EPIs dentro de um programa de proteção dos riscos dos agrotóxicos: qual é a real proteção oferecida por estes equipamentos? Outra linha de pesquisa é o desenvolvimento de EPIs com materiais que, além de proteger para o risco dos agrotóxicos, apresentem conforto térmico, adequado ao clima tropical, dentro de um nível razoável de custo, facilitando a adesão ao uso.

\section{O modelo de produção agrícola}

Em uma agenda de pesquisa inovadora sobre Modelo de Desenvolvimento, Agrotóxicos e Saúde, bem como num programa de SST, a principal medida de proteção coletiva seria promover a redução do consumo de agrotóxicos. Considerando o atual modelo de produção agrícola com consumo intensivo e/ou abusivo, a troca de tipos químicos para produtos de menor toxicidade poderia reduzir os casos de intoxicações (KEIFER; GASPERINI; ROBSON, 2010), sendo uma possível abordagem inicial. Além disso, mudanças nas práticas de aplicação de agrotóxicos, incluindo equipamentos, acrescida de restrições ao uso indiscriminado, poderiam reduzir casos de contaminações e custo, seja por desperdício ou pelo uso de produtos inadequados (GARCIA; ALVES FILHO, 2005), isso sem mudar o atual modelo de produção.

Em outra direção, a pesquisa agrícola poderia ser direcionada a subsidiar propostas de modelos sustentáveis de produção agrícola (CARMO, 1998). Muitas controvérsias envolvem esta linha de propostas, começando pelo entendimento do que seria um modelo de agricultura sustentável. Existem propostas que incluem a utilização de transgênicos nos modelos de agricultura sustentável (CORDEIRO, 
2000; PATERNIANI, 2001), gerando grande polêmica (LACEY, 2007), inclusive porque tem sido observado o aumento no consumo de agrotóxicos em lavouras com uso de transgênicos. Historicamente, a agricultura sustentável tem sido identificada com uma abordagem ecológica (CARMO, 1998; COSTA, 2010). Um grande desafio seria garantir o abastecimento de alimentos para toda população.

O Manejo Integrado de Pragas (MIP) integra a proposta de agricultura sustentável, com a adoção de métodos não químicos ou alternativos, tais como feromônios, controle biológico, erradicação de hospedeiros alternativos e retirada das partes vegetais afetadas. A proposta tem sido defendida por profissionais e pesquisadores (HEINRICHS, 2005), embora existam algumas controvérsias (KOGAN; BAJWA, 1999). Assim, avaliação do impacto do MIP no nível contaminação ambiental e humana também deveria ser tema de pesquisa nesta agenda.

A participação do Brasil no cenário mundial como importante produtor de alimentos tem se caracterizado por crescente produtividade, com monoculturas extensas, na agricultura familiar e na patronal. O problema é que a monocultura favorece o crescimento das chamadas pragas agrícolas, o que, por sua vez, pressiona o aumento do uso de agrotóxicos. Assim, o que está em discussão é o modelo

\section{Referências}

ALVES FILHO, J. P. Medidas individuais de proteção no trabalho com agrotóxicos: indicações básicas e limitações. In: SIMPÓSIO INTERNACIONAL DE TECNOLOGIA DE APLICAÇÃO DE AGROTÓXICOS: EFICIÊNCIA, ECONOMIA E PRESERVAÇÃO DA SAÚDE HUMANA E DO AMBIENTE, 2., 2001, Jundiaí. Anais... Campinas: Instituto Agronômico de Campinas, 2001. p. 1-8.

AGÊNCIA NACIONAL DE VIGILÂNCIA SANITÁRIA. Programa de análise de resíduos de agrotóxicos em alimentos - PARA: relatório de atividades do PARA de 2010. Brasília: Anvisa, 2011. Disponível em: <http://portal.anvisa.gov.br/wps/wcm/ connect/b380fe004965d38ab6abf74ed75891ae/ Relat\%C3\%B3rio+PARA+2010++ Vers\%C3\%A3o+Final.pdf?MOD=AJPERES $>$. Acesso em: 31 maio 2012.

ARAUJO, A. J. et al. Exposição múltipla a agrotóxicos e efeitos à saúde: estudo transversal em amostra de 102 trabalhadores rurais, Nova Friburgo, RJ. Ciência \& Saúde Coletiva, Rio de Janeiro, v. 12, n. 1, p. 115-130, jan./mar. 2007.

ARCURY, T. A. et al. Seasonal variation in the measurement of urinary pesticide metabolites among Latino farmworkers in eastern North de produção de forma ampla, que envolve mudanças conceituais e das práticas agrícolas.

Este tema deve ser pauta obrigatória de pesquisas e de debates com a sociedade brasileira para definir qual o caminho da sustentabilidade e em quem ritmo deve ser trilhado. Além disso, necessariamente, esta discussão deve implicar em um redirecionamento da formação dos profissionais da área agrícola. Este campo de atuação, portanto, também deveria estar contemplado nas linhas de ação e pesquisa. Levando em conta os interesses conflitantes que envolvem este tema, a transição para um modelo de produção agrícola sustentável deve ser conduzida com apoio da maioria da população brasileira e de seus representantes públicos. Enquanto isso, o desafio imediato é proteger a saúde das pessoas, especialmente dos que trabalham expostos a esta enorme carga química.

Na verdade, os desafios são muitos, mas, diante da dimensão dos riscos para a saúde e o meio ambiente decorrentes da intensa exposição aos agrotóxicos, devem ser definidas prioridades e estratégias de ação. Uma agenda de pesquisas e políticas de saúde visando a um desenvolvimento sustentável deve buscar uma integração transversal de vários setores da saúde coletiva, da educação e do setor agrícola, buscando proteger a saúde dos trabalhadores e da população brasileira.

Carolina. International Journal of Occupational and Environmental Health, v. 15, n. 4, p. 339-350, Oct./Dec. 2009.

. Repeated pesticide exposure among North

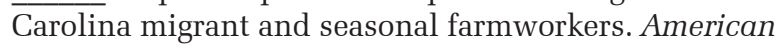
Journal Industrial Medicine, v. 53, n. 8, p. 802-813, Aug. 2010.

. Farmworker exposure to pesticides: methodologic issues for the collection of comparable data. Environmental Health Perspectives, v. 114, n. 6, p. 923-928, Jun. 2006.

BALDI, I. et al. Pesticide contamination of workers in vineyards in France. Jurnal of Exposure Science and Environmental Epidemiology, v. 16, n. 2, p. 115-124, Mar. 2006.

BRASIL. Ministério da Saúde. Coordenação-Geral de Vigilância em Saúde Ambiental. Informe unificado das informações sobre agrotóxicos existentes no SUS - Dados e indicadores selecionados, 2008. v. 2. Disponível em: < http://portal.saude.gov.br/portal/ arquivos/pdf/folder_cgvam_agrotoxicos_2008.pdf $>$. Acesso em: 18 de jull. 2011.

. Ministério da Agricultura Pecuária e Abastecimento. Assessoria de Gestão estratégica. Projeções do Agronegócio 2010/2011 a 2020/2021. 2011a. 
Disponível em: < http://www.agricultura.gov.br/arq editor/file/Ministerio/gestao/projecao/PROJECOES\%20 DO\%20AGRONEGOCIO\%202010-11\%20a\%20202021\%20-\%202_0.pdf >. Acesso em: 24 jul. 2011.

. Ministério da Saúde. Portaria 104 de 25 de janeiro de 2011. Brasília, DF: Ministério da Saúde, 2011b. Disponível em: <http://bvsms.saude.gov.br/ bvs/saudelegis/gm/2011/prt0104_25_01_2011.html >. Acesso em: 22 jul. 2011.

BENTO GONÇALVES [Município]. Secretaria de Vigilância Epidemiológica. Relatório epidemiológico - intoxicações humanas, sistema de informações sobre intoxicações - Sinintox-BG, 1998-2008. Bento Gonçalves: Secretaria Municipal de Saúde - Serviço de Vigilância Epidemiológica, 2009.

CARDENAS, O. et al. Estudio epidemiologico de exposicion a plaguicidas organofosforados y carbamatos en siete departamentos colombianos, 1998-2001. Biomédica, v. 25, n. 2, p. 170-180, jun. 2005.

CARMO, M. S. A Produção familiar como locus ideal da agricultura sustentável. Agricultura em São Paulo, São Paulo, v. 45, n. 1, p.1- 15, 1998.

CATANO, H. C. et al. Plasma cholinesterase levels and health symptoms in peruvian farm workers exposed to organophosphate pesticides. Archives of Environmental Contamination and Toxicology, v. 55, n. 1, p. 153-9, jul. 2008.

CORDEIRO, A. R. Plantas transgênicas: o futuro da agricultura sustentável. História, Ciências, SaúdeManguinhos, Rio de Janeiro, v. 7, n. 2, p. 499-502, jul./ ago. 2000 .

COSTA, A. A. V. M. R. Agricultura sustentável I: conceitos. Revista de Ciências Agrárias, v. 33, n. 2 p. 61-74, 2010.

CURWIN, B. D. et al. Urinary pesticide concentrations among children, mothers and fathers living in farm and non-farm households in Iowa. Annal of Occupational Hygiene, v. 51, n. 1, p. 53-65, Jan. 2007.

FARAHAT, F. M. et al. Chlorpyrifos exposures in Egyptian cotton field workers. Neurotoxicology, v. 31, n. 3, p. 297-304, Jun. 2010.

FARIA, N. M. X. et al. Trabalho rural e intoxicações por agrotóxicos. Cadernos de Saúde Pública, v. 20, n. 5, p. 1298-308, set./out. 2004.

FARIA, N. M. X.; FASSA, A. G.; FACCHINI, L. A. Intoxicação por agrotóxicos no Brasil: os sistemas oficiais de informação e desafios para realização de estudos epidemiológicos. Ciência \& Saúde Coletiva, Rio de Janeiro, v. 12, n. 1, p. 25-38, jan./mar. 2007.

FARIA, N. M. X.; ROSA, J. A. R.; FACCHINI, L. A. Intoxicações por agrotóxicos entre trabalhadores rurais de fruticultura, Bento Gonçalves, RS. Revista de Saúde Pública, São Paulo, v. 43, n. 2, p. 335-44, abr. 2009.

FUSTINONI, S. et al. Biological monitoring and questionnaire for assessing exposure to ethylenebisdithiocarbamates in a multicenter European field study. Human \& Experimental Toxicology, v. 27, n. 9, p. 681-691, Sept. 2008.

GARCIA, E. G.; ALVES FILHO, J. P. Aspectos de prevenção e controle de acidentes no trabalho com agrotóxicos. São Paulo: Fundacentro, 2005.

HEINRICHS, E. A. A new paradigm for implementing ecologically - based participatory IPM in a global context: the IPM CRSP model. Neotropical Entomology, v. 34, n. 2, p. 143-153, Mar./Apr. 2005.

INSTITUTO BRASILEIRO DE GEOGRAFIA E ESTATÍSTICA. Sistema de Recuperação Automática - SIDRA. Censo agropecuário 2006. IBGE, 2006. Disponível em: <http://www.sidra.ibge.gov.br/bda/ tabela/listabl.asp?z=t\&c=913>. Acesso em: 15 jul. 2011.

JONES, K. et al. Determination of ethylenethiourea in urine by liquid chromatography-atmospheric pressure chemical ionisation-mass spectrometry for monitoring background levels in the general population. Journal of Chromatography B: Biomedical Sciences and Applications, v. 878, n. 27, p. 2563-2566, Oct. 2009.

JUREWICZ, J.; HANKE, W. Exposure to pesticides and childhood cancer risk: has there been any progress in epidemiological studies? International Journal of Occupational Medicine and Environmental Health, v. 19, n. 3, p. 152-169, 2006.

KEIFER, M.; GASPERINI, F.; ROBSON, M. Pesticides and other chemicals: minimizing worker exposures. Journal of Agromedicine, v. 15, n. 3, p. 264-274, Jul. 2010.

KEIFER, M. C.; FIRESTONE, J. Neurotoxicity of pesticides. Journal of Agromedicine, v. 12, n. 1, p. 1725. 2007.

KISSMANN, K. G. Rumos e tendências da pesquisa em tecnologia de aplicação de agrotóxicos: A visão da indústria química. In: SIMPÓSIO INTERNACIONAL DE TECNOLOGIA DE APLICAÇÃO DE AGROTÓXICOS: EFICIÊNCIA, ECONOMIA E PRESERVAÇÃO DA SAÚDE HUMANA E DO AMBIENTE, 2., 2001, Jundiaí. Anais... Campinas: Insituto Agronômico de Campinas, 2001.

KOGAN, M.; BAJWA, W. I. Integrated Pest Management: a global reality? Anais da Sociedade Entomológica do Brasil, v. 28, n. 1, p. 1-25. 1999.

LACEY, H. Há alternativas ao uso dos transgênicos? Novos Estudos CEBRAP, n.78, p. 31-39, 2007.

Disponível em: < http://www.scielo.br/pdf/nec/n78/05. pdf $>$. Acesso em: 25 jul. 2011.

LU, C. et al. A longitudinal approach to assessing urban and suburban children's exposure to pyrethroid pesticides. Environmental Health Perspectives, v. 114, n. 9, p. 1419-1423, Sept. 2006. 
NGO, M. A.; O’MALLEY, M.; MAIBACH, H. I.

Percutaneous absorption and exposure assessment of pesticides. Journal of Applied Toxicology, v. 30, n. 2, p. 91-114, Mar. 2010.

OLIVEIRA-SILVA, J. J.; MEYER, A. O Sistema de notificação das intoxicações: o fluxograma da joeira. In: PERES, F.; MOREIRA, J. C. (Ed.). É veneno ou é remédio? Agrotóxicos, saúde e meio ambiente. Rio de janeiro: Fiocruz, 2003. p. 317-326.

OLIVEIRA, M. L.; MACHADO NETO, J. G. Segurança na aplicação de agrotóxicos em cultura de batata em regiões montanhosas. Revista Brasileira de Saúde Ocupacional, São Paulo, v. 30, n. 112, p. 15-25, 2005.

PATERNIANI, E. Agricultura sustentável nos trópicos. Estudos Avançados, São Paulo, v. 15, n. 43, p. 303-326, 2001.

PERRY, M. J.; MARBELLA, A.; LAYDE, P. M. Nonpersistent pesticide exposure self-report versus biomonitoring in farm pesticide applicators. Annals of Epidemiology, v. 16, n. 9, p. 701-707, Sept. 2006.

RAY, D. E.; RICHARDS, P. G. The potential for toxic effects of chronic, low-dose exposure to organophosphates. Toxicology Letters, v. 120, n. 1-3, p. 343-351, Mar. 2001.
RIOS, B. J.; SOLARI, G. S. Biomonitorizacion de plaguicidas: una necesidad del pais? Revista Médica de Chile, v. 138, n. 4, p. 515-518, Apr. 2010.

SOARES, W. L.; FREITAS, E. A. V.; COUTINHO, J. A. G. Trabalho rural e saúde: intoxicações por agrotóxicos no município de Teresópolis - RJ. Revista de Econonomia de Sociologia Rural, v. 43, n. 4, p. 685701, 2005.

THOMAS, K. W. et al. Urinary biomarker, dermal, and air measurement results for 2,4-D and chlorpyrifos farm applicators in the agricultural health study. Jurnal of Exposure Science and Environmental Epidemiology, v. 20, n. 2, p. 119-134, Mar. 2010.

THUNDIYIL, J. G. et al. Acute pesticide poisoning: a proposed classification tool. Bulletin World Health Organization, v. 86, n. 3, p. 205-209, Mar. 2008.

VEIGA, M. M. et al. A contaminação por agrotóxicos e os Equipamentos de Proteção Individual (EPIs).

Revista Brasileira de Saúde Ocupacional, São Paulo, v. 32, n. 116, p. 57-68, 2007.

VITALI, M. et al. Operative modalities and exposure to pesticides during open field treatments among a group of agricultural subcontractors. Archives of Environmental Contamination and Toxicology, v. 57, n. 1, p. 193-202, Jul. 2009.

Recebido: 15/08/2011

Aprovado: 27/04/2012 